\title{
APPLICATION THE SPARSE MATRIX METHOD TO CALCULATE THE METAL ELASTIC STRESS-STRAIN STATE, USING THE FINITE ELEMENT METHOD
}

\author{
Inna I. Barankova ${ }^{1}$, Uliana V. Mikhailova ${ }^{1}$, Victor B. Demidovich ${ }^{2}$ \\ ${ }^{1}$ Nosov Magnitogorsk State Technical University (NMSTU) \\ 38 Lenina str, Magnitogorsk, 455000, Russian Federation \\ e-mail: inna_barankova@mail.ru, ylianapost@gmail.com \\ ${ }^{2}$ Sankt-Petersburg Electrotechnical University (LETI) \\ 5 Prof.Popov str, St.Petersburg, 197376, Russian Federation \\ e-mail: vbdemidovich@mail.ru
}

Keywords: sparse matrix, elastic stress-strain state, compliance, solid mechanics.

\begin{abstract}
The metal stress-strain state simulation needs high precision computations. The use of any simplifying assumptions, such as the plane section hypothesis, the deformable material relative compressibility, uniform temperature distribution over the cross and longitudinal sections of the strip, etc. reduces the computation accuracy of strain and stress at the nodes. Standard programs often use such assumptions. It is acceptable for solving the routine problems. For the manufacture of critical elements such assumptions can lead to undesirable serious consequences. Modeling of the metal forming processes by using the finite element method gives a more accurate solution of the problem. However, the solutions require to solve a linear equations system of high dimensionality. The coefficient matrix is sparse, namely it consists of mainly zero elements, randomly scattered. This leads to considerable computation time for solution methods. In general, the number of arithmetic operations is proportional to the cube of the system's order. In this paper, we propose to increase the computational efficiency analysis through the use of the high-sparse matrix properties. In this case computer time will be proportional to the system order to the 1.2 power. The proposed solution SLAE comprises sparse matrix methods, which include techniques for optimal ordering of matrix rows and columns by using the criterion of the rows in order to increase the first non-zero elements number. This paper shows the application of the computation method for the process of the Belleville spring upset. Belleville springs can be classified as critical elements. One of its possible applications is the rail joint. Unlike conventional parts the elastic elements are characterized by the presence of large deformation under loads. The main factor that determines the service properties of the elastic element is the amount of strain energy that it can be accumulated. Accumulation and dispersing of the Belleville springs strain energy is directly depends to the relation of the meridian cross-section size and the spring working area. The establishment of this dependence is necessary for its rational design.
\end{abstract}




\section{INTRODUCTION}

The metal stress-strain state computation is widely used in the metal forming processes simulation, as well as for the investigation of finished product fatigue strain. The study of stresses and strains arising during the various process stages, will improve the manufacturing process of metal products. Simulation identifies areas with critical stress. Then through the modernization of stamping tools and products specifications arising stresses will be reduced in these areas. This in turn will lead to increase the lifetime of the stamping equipment and manufactured metal products. To obtain the required results, numerical methods should be used.

Consideration of the historical development of numerical methods for the metal stressstrain state computation, shows the gradual improvement of these methods. The purpose of this is to improve the any task computations accuracy in any conditions. To achieve this, first of all it is necessary to provide a solution to the problem without the plane section hypothesis, the deformable material relative compressibility, uniform temperature distribution over the cross and longitudinal sections of the strip, etc. In plastic metal working modeling by using the finite element method the majority of simplifying assumptions is no need to use. In most FEM codes usage of the computer methods the stress-strain state is based on the variational computation, created by O.C. Zienkiewiczand R.C. Taylor[1], R. Hill [2]-[4], D.H. Norrieand G. deVries [5], D.Banabic [6], F. Barlat [7]-[12], J.L. Bassani [13], et al. However, the use of FEM codes for irregular shaped parts requires considerable computer resources. One possible solution to this problem is the $2 \mathrm{D}$ model prediscovery and then on the problem areas more accurate 3D simulation [14].

While there is great diversity in the formulations of the FEM, in general the method can be characterized by the following properties:

1. The task physical area is divided into sub-areas, or finite elements.

2. The dependent variable is approximated by the specialized functions on each finite element and, consequently, in the entire region. The parameters of these approximations in the subsequent are unknown the problem parameters.

3. Substitution approximations to defining equation gives the equations set system with unknown parameters. Solving these equations, we can determine the values of these parameters and, consequently, obtain an approximate the problem solution.

As a result of solving the SLAE for the elastic stress-strain state computation is obtained stress and strain in each finite element. However, the solutions require to solve a linear equations system of high dimensionality. The coefficient matrix is sparse, namely it consists of mainly zero elements, randomly scattered. This leads to considerable computation time for solution methods. In general, the number of arithmetic operations is proportional to the cube of the system's order. In this paper, we propose to increase the computational efficiency analysis through the use of the high-sparse matrix properties. This computer time will be proportional to the system order to the 1.2 power. The proposed solution SLAE comprises sparse matrix methods, which include techniques for optimal ordering of matrix rows and columns by using the criterion of the rows in order to increase the first non-zero elements number.

This paper shows the application of the numerical method for the process of the Belleville spring upset. One of its possible applications in the rail joint. Belleville springs are also commonly used in engineering for damping dynamic and shock loads encountered in operation of machines (anti-vibration damper and other buffers). So the Belleville springs can be classified as critical elements. However, according to numerous studies and practical testing of a significant portion of the Belleville springs during exploitation under repeated 
loading does not stand above the claimant the amount of cycles $\left(2 \cdot 10^{6}\right)$. Damage occurs due to development of fatigue cracks in the edge hole due to wear of their contact surfaces. Defects in the Belleville spring edges surface, appearing in the typical stamping and accelerates the fatigue cracks nucleation. In standard Belleville springs during operation there are significant the stresses value, and this their service life is reduced. Contact spring surface wears out quickly due to its small area. To increase the spring service life necessary to reduce the stresses during exploitation strain, to increase the contact surface area and eliminate the occurrence of surface defects during stamping. Reducing stress possible by optimizing the design parameters of the spring based on the stress-strain state analysis results of its compression during use [15].

\section{APPLICATION OF THE NUMERICAL METHODS TO ANALYSIS THE STRESS-STRAIN STATE}

\subsection{Application of the finite element method to compute the stress-strain state problems}

Consider the application of finite element method for solving the computer problem the Belleville spring elastic stress-strain state. The computer problem the spring elastic stressstrain state resolved on the basis of the minimum potential energy principle [5], [16]. The potential energy functional for solid mechanics bidimensional problems looks as follows:

$$
\chi=\int_{V} \frac{1}{2} \varepsilon^{T} \sigma d V-\int_{V} U^{T} P d V-\int_{A_{T}} U^{T} T d A_{T},
$$

where $\varepsilon=\left[\begin{array}{c}\varepsilon_{x} \\ \varepsilon_{y} \\ \gamma_{x y}\end{array}\right]$ - the strain vector, $\sigma=\left[\begin{array}{c}\sigma_{x} \\ \sigma_{y} \\ \tau_{x y}\end{array}\right]-$ the stress vector, $U^{T}-$ the transpose $U=\left[\begin{array}{l}u \\ v\end{array}\right]$ of the movement at point matrix, $\mathrm{P}=\left[\begin{array}{l}\mathrm{P}_{x} \\ \mathrm{P}_{y}\end{array}\right]$ - the mass force per unit volume matrix, $T=\left[\begin{array}{l}T_{x} \\ T_{y}\end{array}\right]$ - surface stress at point matrix, $V$ - solid volume, $A_{T}$-solid surface.

Values $\varepsilon_{x}, \varepsilon_{y}$ and $\sigma_{x}, \sigma_{y}$ are strains and stresses axial $x$ and $y$, respectively. In constructing a mathematical model of the elastic stress-strain state in a spring-loaded by the linearity of the elastic problem and small strain velocities do not take into account the temperature changes effects and the mass forces emergence [16]. These factors can be taken into account additionally if necessary.

Consequently:

$$
\begin{gathered}
F=0 \\
\delta T=0,
\end{gathered}
$$

where $F$-mass force potential, $\delta T$ - solid temperature increment.

Due to the fact that the Belleville spring is in equilibrium, and consider strain state, the true system of solid material points displacement $u$ by the loads action $T$, as well as taking into account the condition (2), the potential energy functional (1) transform as follows:

$$
\chi=\frac{1}{2} \int_{V} \varepsilon \sigma d V-\int_{A_{T}} u T d A_{T}
$$

where $V$ - solid volume, $T=\left[\begin{array}{l}T_{x} \\ T_{y}\end{array}\right]$ - surface stress vector, $A_{T}-$ outer contact surface. 
Since the Belleville spring has a rotation solid shape (Figure 1), then the decision we consider axisymmetrical stress-strain state.

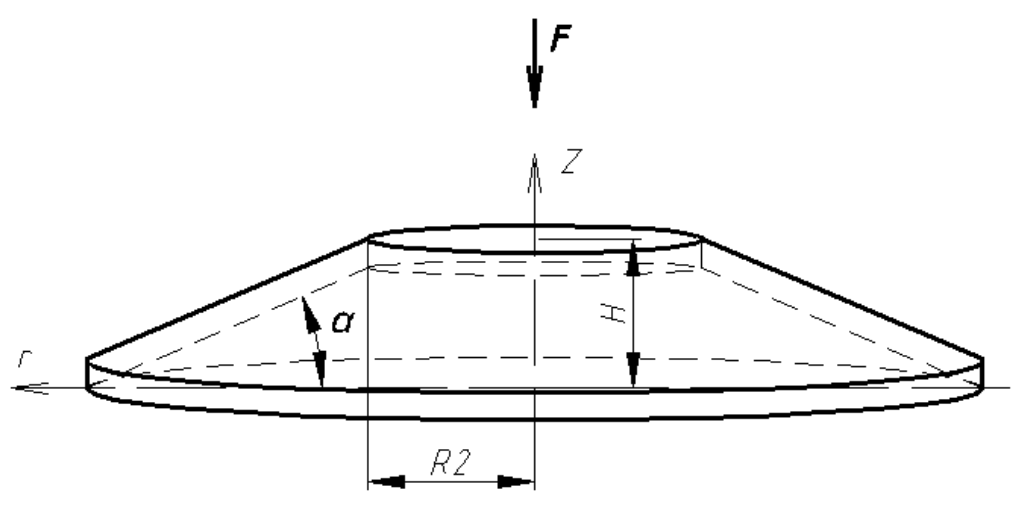

Figure 1: Parameters Belleville spring and the cylindrical system position.

After the conversion, taking into account characteristics of the Belleville spring parameters, the potential energy functional (3) for the spring will be:

$$
\chi=\frac{1}{2} \int_{0}^{2 \pi} d \varphi \int_{R_{2}}^{R_{1}} \int_{z_{2}}^{z_{1}} \varepsilon^{T} \cdot D \cdot \varepsilon \cdot r \cdot d r d z-k \cdot p_{z} \cdot \delta \int_{0}^{2 \pi} R_{2} \cdot u d \varphi
$$

where $z_{1}=H_{1}-\frac{H_{1}}{R_{1}} r ;, z_{2}=H_{2}-\frac{H_{1}}{R_{1}} r, H_{1}=R_{1} \cdot \operatorname{tg} \alpha, H_{2}=H_{1}+\frac{S}{\cos \alpha}, H-$ the Belleville spring height, $k$ - friction coefficient, $R_{1}$ и $R_{2}$ - the Belleville spring radius and spring holes, respectively (Figure 1).

Then displacement to each of the finite element is approximated by linear functions. Then using the differentiation matrix rules, we compute the partial derivatives of the elemental energies along the nodal displacements vectors. The Belleville spring cross section break in the 48 triangular elements. Then, after securing the condition of potential energy functional minimum (4) and implement the united elemental matrix equations (association for nodes) in the matrix equation system with the defined movement. A result we get linear equations system for the nodal displacements values:

$\sum_{e=1}^{48} K^{e} U^{e}-\frac{F \cdot S \cdot\left(R_{1}-R_{2}\right)}{288 \cdot \pi \cdot R_{2} \cdot \cos \alpha}=0$,
where $K^{e}=120,859 \cdot S\left(R_{1}-R_{2}\right) \cdot\left(\begin{array}{lllllll}\kappa_{11} & \kappa_{12} & \kappa_{13} & \kappa_{14} & \kappa_{15} & \kappa_{16} \\ \kappa_{21} & \kappa_{22} & \kappa_{23} & \kappa_{24} & \kappa_{25} & \kappa_{26} \\ \kappa_{31} & \kappa_{32} & \kappa_{33} & \kappa_{34} & \kappa_{35} & \kappa_{36} \\ \kappa_{41} & \kappa_{42} & \kappa_{43} & \kappa_{44} & \kappa_{45} & \kappa_{46} \\ \kappa_{51} & \kappa_{52} & \kappa_{53} & \kappa_{54} & \kappa_{55} & \kappa_{56} \\ \kappa_{61} & \kappa_{62} & \kappa_{63} & \kappa_{64} & \kappa_{65} & \kappa_{66}\end{array}\right)$, 


$$
\begin{aligned}
& \text { where } \kappa_{i 1}=\left(\begin{array}{c}
\left(z_{2}-z_{3}\right)^{2}+0,35 \cdot\left(r_{3}-r_{2}\right)^{2} \\
0,3 \cdot\left(r_{3}-r_{2}\right)\left(z_{2}-z_{3}\right)+0,35 \cdot\left(z_{2}-z_{3}\right)\left(r_{3}-r_{2}\right) \\
\left(z_{3}-z_{1}\right)\left(z_{2}-z_{3}\right)+0,35 \cdot\left(r_{1}-r_{3}\right)\left(r_{3}-r_{2}\right) \\
0,3 \cdot\left(r_{1}-r_{3}\right)\left(z_{2}-z_{3}\right)+0,35 \cdot\left(z_{3}-z_{1}\right)\left(r_{3}-r_{2}\right) \\
\left(z_{1}-z_{2}\right)\left(z_{2}-z_{3}\right)+0,35 \cdot\left(r_{2}-r_{1}\right)\left(r_{3}-r_{2}\right) \\
0,3 \cdot\left(r_{2}-r_{1}\right)\left(z_{2}-z_{3}\right)+0,35 \cdot\left(z_{1}-z_{2}\right)\left(r_{3}-r_{2}\right)
\end{array}\right) ; \quad \kappa_{i 2}=\left(\begin{array}{c}
0,3 \cdot\left(r_{3}-r_{2}\right)\left(z_{2}-z_{3}\right)+0,35 \cdot\left(z_{2}-z_{3}\right)\left(r_{3}-r_{2}\right) \\
0,35 \cdot\left(z_{2}-z_{3}\right)^{2}+\left(r_{3}-r_{2}\right)^{2} \\
0,3 \cdot\left(z_{3}-z_{1}\right)\left(r_{3}-r_{2}\right)+0,35 \cdot\left(r_{1}-r_{3}\right)\left(z_{2}-z_{3}\right) \\
\left(r_{1}-r_{3}\right)\left(r_{3}-r_{2}\right)+0,35 \cdot\left(z_{3}-z_{1}\right)\left(z_{2}-z_{3}\right) \\
0,3 \cdot\left(z_{1}-z_{2}\right)\left(r_{3}-r_{2}\right)+0,35 \cdot\left(r_{2}-r_{1}\right)\left(z_{2}-z_{3}\right) \\
\left(r_{2}-r_{1}\right)\left(r_{3}-r_{2}\right)+0,35 \cdot\left(z_{1}-z_{2}\right)\left(z_{2}-z_{3}\right)
\end{array}\right) \text {; } \\
& \kappa_{i 3}=\left(\begin{array}{c}
\left(z_{3}-z_{1}\right)\left(z_{2}-z_{3}\right)+0,35 \cdot\left(r_{1}-r_{3}\right)\left(r_{3}-r_{2}\right) \\
0,3 \cdot\left(z_{3}-z_{1}\right)\left(r_{3}-r_{2}\right)+0,35 \cdot\left(r_{1}-r_{3}\right)\left(z_{2}-z_{3}\right) \\
\left(z_{3}-z_{1}\right)^{2}+0,35 \cdot\left(r_{1}-r_{3}\right)^{2} \\
0,3 \cdot\left(r_{1}-r_{3}\right)\left(z_{3}-z_{1}\right)+0,35 \cdot\left(z_{3}-z_{1}\right)\left(r_{1}-r_{3}\right) \\
\left(z_{1}-z_{2}\right)\left(z_{3}-z_{1}\right)+0,35 \cdot\left(r_{2}-r_{1}\right)\left(r_{1}-r_{3}\right) \\
0,3 \cdot\left(r_{2}-r_{1}\right)\left(z_{3}-z_{1}\right)+0,35 \cdot\left(z_{1}-z_{2}\right)\left(r_{1}-r_{3}\right)
\end{array}\right) ; \kappa_{i 4}=\left(\begin{array}{c}
0,3 \cdot\left(r_{1}-r_{3}\right)\left(z_{2}-z_{3}\right)+0,35 \cdot\left(z_{3}-z_{1}\right)\left(r_{3}-r_{2}\right) \\
\left(r_{1}-r_{3}\right)\left(r_{3}-r_{2}\right)+0,35 \cdot\left(z_{3}-z_{1}\right)\left(z_{2}-z_{3}\right) \\
0,3 \cdot\left(r_{1}-r_{3}\right)\left(z_{3}-z_{1}\right)+0,35 \cdot\left(z_{3}-z_{1}\right)\left(r_{1}-r_{3}\right) \\
0,35 \cdot\left(z_{3}-z_{1}\right)^{2}+\left(r_{1}-r_{3}\right)^{2} \\
0,3 \cdot\left(z_{1}-z_{2}\right)\left(r_{1}-r_{3}\right)+0,35 \cdot\left(r_{2}-r_{1}\right)\left(z_{3}-z_{1}\right) \\
\left(r_{1}-r_{3}\right)\left(r_{2}-r_{1}\right)+0,35 \cdot\left(z_{1}-z_{2}\right)\left(z_{3}-z_{1}\right)
\end{array}\right) \\
& \kappa_{i 5}=\left(\begin{array}{c}
\left(z_{1}-z_{2}\right)\left(z_{2}-z_{3}\right)+0,35 \cdot\left(r_{2}-r_{1}\right)\left(r_{3}-r_{2}\right) \\
0,3 \cdot\left(z_{1}-z_{2}\right)\left(r_{3}-r_{2}\right)+0,35 \cdot\left(r_{2}-r_{1}\right)\left(z_{2}-z_{3}\right) \\
\left(z_{1}-z_{2}\right)\left(z_{3}-z_{1}\right)+0,35 \cdot\left(r_{2}-r_{1}\right)\left(r_{1}-r_{3}\right) \\
0,3 \cdot\left(z_{1}-z_{2}\right)\left(r_{1}-r_{3}\right)+0,35 \cdot\left(r_{2}-r_{1}\right)\left(z_{3}-z_{1}\right) \\
\left(z_{1}-z_{2}\right)^{2}+0,35 \cdot\left(r_{2}-r_{1}\right)^{2} \\
0,3 \cdot\left(z_{1}-z_{2}\right)\left(r_{2}-r_{1}\right)+0,35 \cdot\left(z_{1}-z_{2}\right)\left(r_{2}-r_{1}\right)
\end{array}\right) ; \kappa_{i 6}=\left(\begin{array}{c}
0,3 \cdot\left(r_{2}-r_{1}\right)\left(z_{2}-z_{3}\right)+0,35 \cdot\left(z_{1}-z_{2}\right)\left(r_{3}-r_{2}\right) \\
\left(r_{2}-r_{1}\right)\left(r_{3}-r_{2}\right)+0,35 \cdot\left(z_{1}-z_{2}\right)\left(z_{2}-z_{3}\right) \\
0,3 \cdot\left(r_{2}-r_{1}\right)\left(z_{3}-z_{1}\right)+0,35 \cdot\left(z_{1}-z_{2}\right)\left(r_{1}-r_{3}\right) \\
\left(r_{2}-r_{1}\right)\left(r_{1}-r_{3}\right)+0,35 \cdot\left(z_{1}-z_{2}\right)\left(z_{3}-z_{1}\right) \\
0,3 \cdot\left(z_{1}-z_{2}\right)\left(r_{2}-r_{1}\right)+0,35 \cdot\left(z_{1}-z_{2}\right)\left(r_{2}-r_{1}\right) \\
0,35 \cdot\left(z_{1}-z_{2}\right)^{2}+\left(r_{2}-r_{1}\right)^{2}
\end{array}\right) .
\end{aligned}
$$

Coefficient matrix of the linear equations system resulting contains a large number of zero elements (a sparse matrix). Therefore, the use of accurate computation methods (for example, the Gauss method) provides more computing error and has low computing speed. Since they depend on the positioning of columns and rows with null elements. This leads to high computations processing power. To improve the efficiency computations used special methods to transform a sparse coefficient matrix, include a method of arranging the rows and columns, reduce the sparse matrix filling, namely a modified Gauss method that provides a numerical method stability [17]. As a result of its solutions obtained nodal displacements, and then determine the stress and strain in each element [18].

\subsection{Application of the sparse matrix method to compute the SLAE}

Using iterative methods for solving linear systems with sparse matrices is not very effective, as to ensure their convergence necessary to satisfy the convergence conditions, in addition, if the problem solving linear systems is incorrect, then the iterative methods do not give a clear answer. Exact methods devoid of these shortcomings, however, their use gives greater computational errors and a higher dimension takes more memory [19]. The errors are caused by the transition from the mathematical model to the numerical method. They are connected with the fact that every numerical method reproduces the original mathematical model approximately. To improve the efficiency computations, there are special methods to transform a sparse coefficient matrix, including the optimal ways to organize the sparse matrix rows and columns.

The Gauss method - the successive unknowns elimination method from a equations system [20]. With the unknown $k_{x}$ exclusion, the equations system:

$$
A x=b \text {, }
$$


where all coefficients $a_{i j}$ when $i>k$ and $j>k$ computed by the formula:

$$
a_{i j}=a_{i j}-\frac{a_{i k} a_{k j}}{a_{k k}}
$$

In general, the arithmetic operations numbers to Gaussian solve (5) be proportional to the system order to the 3 power. This leads to considerable cost of computer time. It is possible to improve significantly the computational efficiency of the analysis, if application the property of the high matrix sparseness $A$ to model (5). Sparse matrix processing efficiency is high because it does not require, firstly, conversion of the formula (6), if at least one of the elements $a_{i k}$ or $a_{k j}$ is zero, secondly, the memory cost to store zero elements. Although sparse matrix algorithms are more complex, but as a result the computer time cost will be closer to linear. When using the sparse matrix methods need to take into account the dependence of the computational efficiency of the coefficient matrix submission $A$, more precisely, on the order in which recorded its rows and columns. Sparse matrix methods should include ways to the optimal streamline of rows and columns matrix. It is possible to use several ordering optimality criteria. The simplest of these is the criterion of the rows in order to increase the number of primary non-zero, more complex criteria take into account not only the primary non-zero, but also the emerging secondary non-zero.

To implement the method of sparse matrices it was developed software in the Delphi language. Input data the program are placed in a separate file containing non-zero values of the matrix coefficients in a packed form. The principle of reading the input data is as follows: at the beginning indicates the matrix order, each subsequent line is in the form kind of $a_{1}, a_{2}$, $a_{3} \ldots$, where the first and the third number - a columns number, where the data is non-zero elements, and the second, the fourth number - data is non-zero elements. This type of the data presentation allows the large dimensions matrices can be stored and processed in the computer's memory. In this case gain in storage costs is significant. Using this software allows you to get a solution to a linear equations system with sparse coefficients matrices, to estimate the error and make a conclusion about the decision correctness [21].

\subsection{Application of the method to analysis the stress-strain state in the spring-loaded}

On the basis of the discussed above computer method the mathematical elastic stress-strain state model of the Belleville spring loaded was worked out. For the numerical implementation of the model it was developed software designed to compute strains and stresses in the springloaded. The program was developed in programming environment $\mathrm{C \#} \mathrm{[18].} \mathrm{Figure} 2$ shows the stresses in the Belleville spring-loaded with design parameters: $S=1 \mathrm{~mm}, R I=17.5 \mathrm{~mm}$, $R 2=4.98 \mathrm{~mm}, \alpha=5.47^{\circ}, H=1.2 \mathrm{~mm}$, when a load is applied $F=110 \mathrm{~N}$.

Problem is solved by using a CAE system «ANSYS» to check the model verification (Figure 3).

On the basis of the stress-strain state analysis the computation method of disc springs optimal design parameters and form was worked out. 


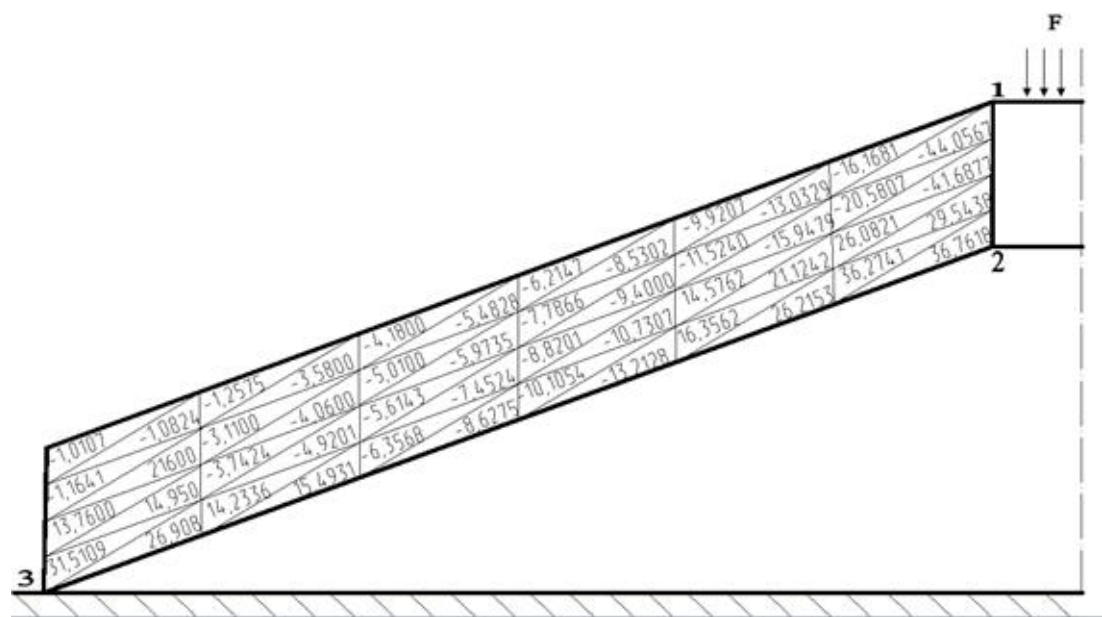

Figure 2: Stresses $\sigma_{r}$ in the Belleville spring-loaded.

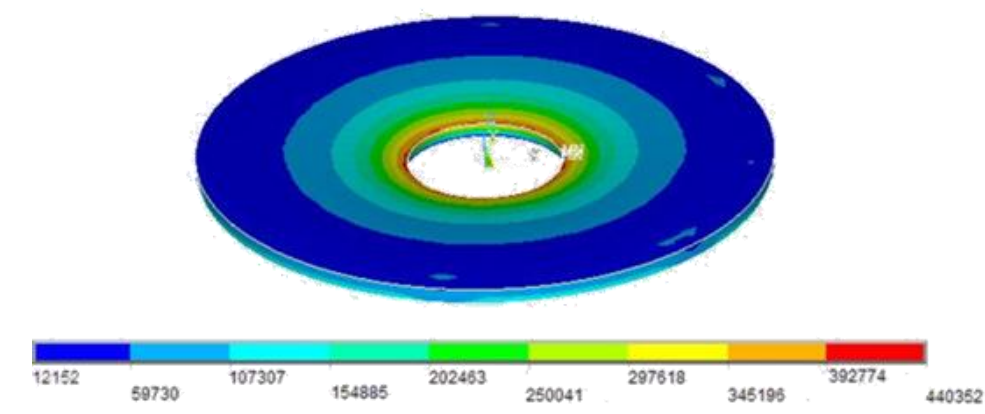

Figure 3: Stresses distribution field in a Belleville spring-loaded.

\section{CONCLUSIONS}

- A mathematical model of elastic springs compression during operation is developed.

- Sparse matrix method is used to improve computation performance.

- For the numerical implementation of the model it was developed software designed to compute strains and stresses in the spring-loaded.

- The stress-strain state of the spring-loaded is analyzed.

- On the basis of the stress-strain state analysis the computation method of optimal springs design parameters and form is worked out.

\section{REFERENCES}

[1] O.C. Zienkiewicz, R.C. Taylor, The finite element method, Vol. I, 4th Edition. McGrawHill, 1989.

[2] R. Hill, A theory of the yielding and plastic flow of anisotropic metals. Proc. R. Soc. Lond. A193, 281-297, 1948.

[3] R. Hill, Theoretical plasticity of textured aggregates. Math.Proc. Cambr. Philos. Soc. 85, 179-191, 1979.

[4] R. Hill, Constitutive modeling of orthotropic plasticity in sheet metals. J. Mech. Phys. Solids 38, 405-417, 1990. 
[5] D.H. Norrie, G. de Vries, An Introduction to Finite Element Analysis. Academic Press, London, 1978.

[6] D. Banabic, H. Aretz, D.S. Comsa, et al., An improved analytical description of orthotropy in metallic sheets. Int. J. Plast. 21, 493-512, 2005.

[7] F. Barlat, J. Lian, Plastic behavior and stretchability of sheet metals. Part I: a yield function for orthotropic sheets under plane stress conditions. Int. J. Plast. 5, 51-66, 1989.

[8] F. Barlat, D.J. Lege, J.C. Brem, A six-component yield function for anisotropic materials. Int. J. Plast. 7, 693-712, 1991.

[9] F. Barlat, Y. Maeda, K. Chung, et al., Yield function development for aluminum alloy sheet. J. Mech. Phys. Solids 45, 1727-1763, 1997.

[10] F. Barlat, J.C. Brem, J.W. Yoon, et al., Plane stress yield function for aluminum alloy sheets. Part I: theory. Int. J. Plast. 19, 1297-1319, 2003.

[11] F. Barlat, H. Aretz, J.W. Yoon, et al., Linear transformation based anisotropic yield functions. Int. J. Plast. 21, 1009-1039, 2005.

[12] F. Barlat, J.W. Yoon, O. Cazacu, On linear transformation of stress tensors for the description of plastic anisotropy. Int. J. Plast. 23, 876-896, 2007.

[13] J.L. Bassani, Yield characterisation of metals with transversally isotropic plastic properties. Int. J. Mech. Sci. 19, 651-660, 1977.

[14] J. Wang, J Sun, Plane strain transversely anisotropic analysis in sheet metal forming simulation using 6-component Barlat yield function. International Journal of Mechanics and Materials in Design, Vol. 8, Issue 4, 327-333, 2012.

[15] U.V. Mikhailova, Improvement technology manufacturing special Belleville spring with elastic medium. Dissertation for the degree of technical sciences candidate. Magnitogorsk, Nosov Magnitogorsk state technical university, 2011.

[16] O.S. Zhelezkov, S.V. Kochukov, U.V. Mikhailova, Modeling compress the Belleville springs by using finite element method. Processes and equipment of metallurgical production. Magnitogorsk, NMSTU, 239-243, 2006.

[17] U.V. Mikhailova, Application of finite element method for the metal stress-strain state computation. Mathematics. Mathematics applications in the economic, technical and pedagogical research. Magnitogorsk, NMSTU, 258-260, 2006.

[18] U.V. Mikhailova, The elastic stress-strain state Belleville spring modeling using the finite element method. The certificate of registration of the branch development № 8128. M., 2007. № 50200700_808.

[19] I.P. Norenkov, Fundamentals of computer-aided design. Moscow, Bauman MSTU, 2002.

[20] R. Tyuarson, Sparse matrix. Moscow, 1977.

[21] G.M. Korinchenko, U.V. Mikhailova, K.A. Kislitsin, Sparse matrix application for the design solution analysis design elements in mechanical engineering. The application of mathematics to economic and technical studies№ 1 (1), Magnitogorsk, NMSTU, 142$145,2007$. 